\title{
Efficacy and Safety of MLC601 in the Treatment of Mild Cognitive Impairment: A Pilot, Randomized, Double-Blind, Placebo-Controlled Study
}

\author{
Hossein Pakdaman Ali Amini Harandi Mehdi Abbasi \\ Hosein Delavar Kasmaei Farzad Ashrafi Koroush Gharagozli \\ Farhad Assarzadegan Behdad Behnam Mehran Arabahmadi \\ Brain Mapping Research Center, Shahid Beheshti University of Medical Sciences, Tehran, Iran
}

\section{Keywords}

Mild cognitive impairment · MLC601 · Placebo-controlled study · Double-blind study · Safety

\begin{abstract}
Background and Aim: Mild cognitive impairment (MCI) is characterized by declined cognitive function greater than that expected for a person's age. The clinical significance of this condition is its possible progression to dementia. MLC601 is a natural neuroprotective medication that has shown promising effects in Alzheimer disease. Accordingly, we conducted this randomized, double-blind, placebo-controlled study to evaluate the efficacy and safety of MLC601 in MCI patients. Methods: Seventy-two patients with a diagnosis of MCI were recruited. The included participants were randomly assigned to groups to receive either MLC601 or placebo. An evaluation of global cognitive function was performed at baseline as well as at 3-month and 6-month follow-up visits. Global cognitive function was assessed by MiniMental State Examination (MMSE) and Alzheimer's Disease Assessment Scale-cognitive subscale (ADAS-cog) scores. Efficacy was evaluated by comparing global function scores between the 2 groups during the study period. Safety assessment included adverse events (AEs) and abnormal laboratory results. Results: Seventy patients completed the study, 34 in the MLC601 group and 36 in the placebo group. The mean changes $( \pm S D)$ in cognition scores over 6 months in the MLC601 group were $-2.26( \pm 3.42)$ for the MMSE and $3.82( \pm 6.16)$ for the ADAScog; in the placebo group, they were $-2.66( \pm 3.43)$ for the MMSE and $4.41( \pm 6.66)$ for the ADAS-cog. The cognition changes based on both MMSE and ADAS-cog scores were statistically significant between the placebo and the MLC601 group $(p<0.001)$. Only 5 patients (14.7\%) reported minor AEs in the MLC601 group, the most commonly reported of which were gastrointestinal, none of them leading to patient withdrawal. Conclusion: MLC601 has shown promising efficacy and acceptable AEs in MCI patients.


Pakdaman et al.: Efficacy and Safety of MLC601 in the Treatment of Mild Cognitive Impairment: A Pilot, Randomized, Double-Blind, Placebo-Controlled Study

\section{Introduction}

Mild cognitive impairment (MCI) is a condition defined as declined cognitive function greater than that expected for a person's age and educational level which does not affect an individual's life in a noticeable manner. The prevalence of this syndrome varies between studies, with a reported prevalence of 3-19\% above the age of 65 years [1]. The clinical significance of $\mathrm{MCI}$ is its possible progression to dementia, with an approximately $5-10 \%$ annual conversion rate [2]. The observed discrepancies in MCI prevalence and progression rates between studies are probably caused by controversial debate about the definition used for $\mathrm{MCI}$, since $\mathrm{MCI}$ is a heterogeneous disorder and may progress to Alzheimer disease or to other dementing illnesses (including vascular dementia, frontotemporal dementia, and Lewy body dementia) or remain relatively stable and lead to cognitive decline as in normal aging [3].

Currently, the idea of a continuum of cognitive decline is being supported by the introduction of a "neurocognitive disorder" (NCD) category in the Diagnostic and Statistical Manual of Mental Disorders, 5th edition (DSM-5) [4]. The DSM-5 has expanded its diagnostic category to include MCI (mild NCD in the DSM-5) in an effort to help diagnose NCDs before the beginning of the clinical manifestation of dementia $[5,6]$. Therefore, much more attention should be paid to MCI in aspects important to patients including prevention, treatment, and clinical outcome.

In terms of treatment, a growing body of literature on medical therapy for $\mathrm{MCI}$ has been focusing on using anti-dementia drugs which can slow the rate of cognitive decline; however, their use is limited due to their side effects. MLC601 (NeuroAiD ${ }^{\circledR}$ ) is a natural, neuroprotective medication that in our previous studies on Alzheimer patients had shown promising efficacy with few side effects compared to treatment with standard cholinesterase inhibitors $[7,8]$. Accordingly, we conducted this pilot, randomized, double-blind, placebo-controlled study to evaluate the efficacy and safety of MLC601 in patients with MCI.

\section{Subjects and Methods}

\section{Study Design}

This was pilot, randomized, double-blind, placebo-controlled study aimed at evaluating the efficacy and safety of MLC601 in patients with MCI. After patient selection and screening, the included participants were randomly assigned to groups to receive either MLC601 (NeuroAiD, Moleac Pte. Ltd, Singapore; 0.4 g per capsule) or a placebo capsule 3 times a day, and they were prospectively followed for 6 months. The MLC601 and placebo capsules were visually identical, and the capsule containers were labeled with the name of the study and the allocated number. An expert statistician generated the allocation schedule using a computer-based program, and an allocation ratio of 1:1 was applied. The study profile is shown in Figure 1.

MLC601 contains 5 animal components $(0.0665 \mathrm{~g}$ Hirudo, $0.0285 \mathrm{~g}$ Cornu saigae tataricae, $0.095 \mathrm{~g}$ Mesobuthus martensii, $0.0665 \mathrm{~g}$ Eupolyphaga seu steleophaga, and $0.0285 \mathrm{~g}$ Calculus bovis artifactus) and 9 herbal components (0.114 g radix Paeoniae rubrae, $0.57 \mathrm{~g}$ radix Astragali, 0.114 g radix Salviae miltiorrhizae, $0.114 \mathrm{~g}$ rhizoma Chuanxiong, $0.114 \mathrm{~g}$ radix Angelicae sinensis, 0.114 g radix Polygalae, 0.114 g Prunus persica, 0.114 g Carthamus tinctorius, and $0.114 \mathrm{~g}$ rhizoma Acori tatarinowii). The placebo capsules were filled with flour as an inert chemical, making them indistinguishable from the MLC601 capsules.

The patients, care partners, and all neurologists directly involved in the study were blinded to the intervention during the period of the study. Demographic and clinical information on the patients was recorded at baseline. An evaluation of global cognitive function and a thorough neurological examination were performed by neurologists at baseline and at 
Fig. 1. Study flowchart.

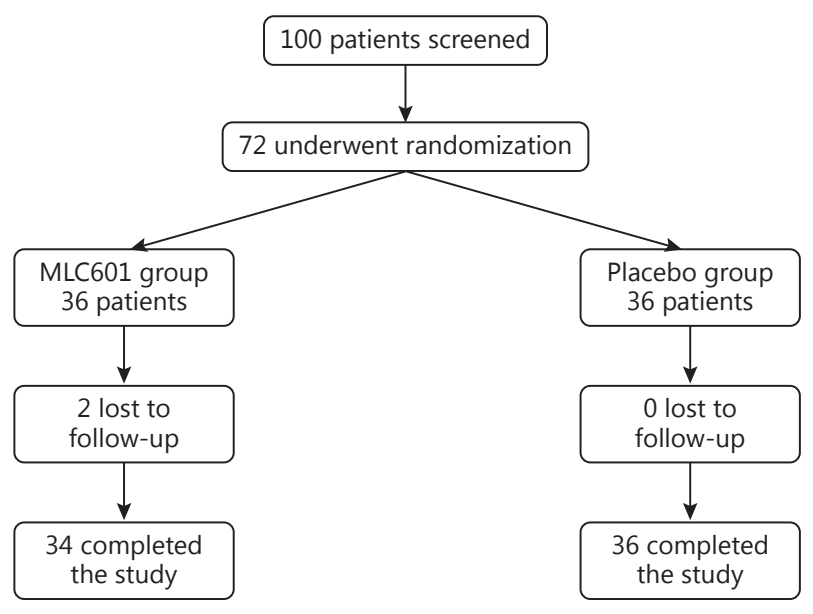

the 3- and 6-month follow-up visits. Global cognitive function was assessed with the MiniMental State Examination (MMSE) and the Alzheimer's Disease Assessment Scale-cognitive subscale (ADAS-cog). The MMSE measures orientation to time (5 points), orientation to place ( 5 points), registration ( 3 points), attention and calculation ( 5 points), recall (3 points), naming and repetition ( 3 points), comprehension ( 3 points), reading ability ( 1 point), writing ability (1 point), and visual construction ( 1 point). Scores range from 0 to 30 (the higher the score, the better the cognitive function) [9]. The standard ADAS-cog includes 7 short cognitive tests and 4 scales rated by a physician, and it ranges from 0 (no impairment) to 70 (severe impairment) [10].

\section{Participants}

Between March 2015 and June 2015, patients with a clinical MCI diagnosis according to Petersen et al. $[11,12]$ were recruited from neurology clinics in Tehran if they were older than 65 years and satisfied the following inclusion criteria: an MMSE score between 23 and 27; an available study partner as an informer; written informed consent signed by the patient and the informer; adequate vision and hearing for neuropsychological testing; a normal vitamin $\mathrm{B}_{12}$ level, thyroid function test result, and nonreactive rapid plasma reagin test result; and a normal electrocardiogram or one with no clinically significant abnormalities. Exclusion criteria were significant cerebral vascular disease (Hachinski ischemic score >4) [13], depression (Hamilton Depression Rating Scale score >12) [14], dementia, receiving antidementia drugs, a clinically significant finding on imaging (infection, infarction, or focal lesion), and severe physical or psychological disease that could interfere with participation in the study (e.g., traumatic brain injury). Patients were considered lost to follow-up if they did not attend the 3-month or the 6-month follow-up visit.

\section{Efficacy Measure}

The efficacy endpoint of the study was to compare the change in cognitive function scores (MMSE and ADAS-cog) between the MLC601 and the placebo group during the study period. 
Table 1. Means and standard deviations of MMSE scores in the placebo and the MLC601 group

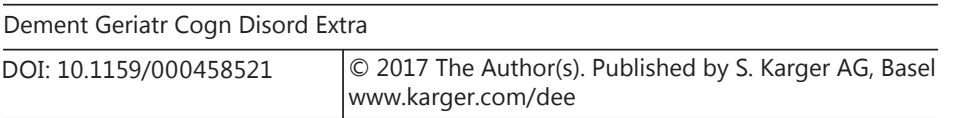

Pakdaman et al.: Efficacy and Safety of MLC601 in the Treatment of Mild Cognitive Impairment: A Pilot, Randomized, Double-Blind, Placebo-Controlled Study

\begin{tabular}{llll}
\hline Group & Baseline & 3 months & 6 months \\
\hline MLC601 & $25.32 \pm 1.41$ & $23.74 \pm 3.17$ & $23.06 \pm 4.03$ \\
Placebo & $25.14 \pm 1.17$ & $23.75 \pm 2.49$ & $22.47 \pm 3.55$ \\
\hline
\end{tabular}

\section{Safety Measure}

Safety was evaluated by recording adverse events (AEs) reported by the participants and their study partners. Additionally, a blood count was conducted and liver and renal function were evaluated on each visit and any abnormality was recorded.

\section{Statistical Analysis}

All statistical analyses were performed by using SPSS 18.0 (SPSS Inc., Chicago, IL, USA). The independent $t$ test, the $\chi^{2}$ test, and the general linear model (repeated measures) were used to interpret data. $p$ values $<0.05$ were considered statistically significant.

\section{Ethical Issues}

The study was designed in accordance with the Declaration of Helsinki and approved by the Ethics Committee at Shahid Beheshti University of Medical Sciences. All patients or their caregivers provided informed consent for participation.

\section{Results}

\section{Baseline Characteristics}

Of 100 interested individuals, 72 patients fulfilled the criteria and were included in the study. Seventy patients completed the study and 2 patients were excluded since they were lost to follow-up. Finally, there were 34 patients in the MLC601 group and 36 patients in the placebo group. The mean (SD) age of the MLC601 and the placebo group was $70.85( \pm 3.92)$ and $70.25( \pm 3.39)$ years, respectively. There was no statistically significant difference between the mean ages of the 2 groups ( $p=0.493$ ).

There were 19 (55.9\%) males in the MLC601 group and 21 (58.3\%) males in the placebo group. The observed difference in gender was not statistically significant ( $p=0.83$ ). Additionally, no significant difference was noticed between the MMSE scores of each group at baseline ( $p=0.553$ ). The difference between the baseline ADAS-cog score of the MLC601 group and that of the placebo group was also not statistically significant ( $p=0.586)$.

\section{Efficacy Outcomes \\ MMSE Score}

The mean MMSE scores and the mean changes from baseline at the 3-and 6-month visits are shown in Table 1 . The mean change $( \pm$ SD) in MMSE score over 6 months for the MLC601 group was $-2.26( \pm 3.42)$, and for the placebo group it was $-2.66( \pm 3.43)$. By the general linear model, repeated-measures analysis was carried out based on the MMSE score, and it showed a statistically significant difference between the placebo and the MLC601 group during the 6 months of follow-up ( $p<0.001)$ (Fig. 2).

\section{ADAS-cog Score}

The mean ADAS-cog scores and the mean changes from baseline at the 3-and 6-month visits are shown in Table 2 . The mean change $( \pm S D)$ in ADAS-cog score over 6 months for the 
Fig. 2. Mean changes in MMSE score from baseline in the placebo and the MLC601 group.

Table 2. Means and standard deviations of ADAS-cog scores in the placebo and the MLC601 group

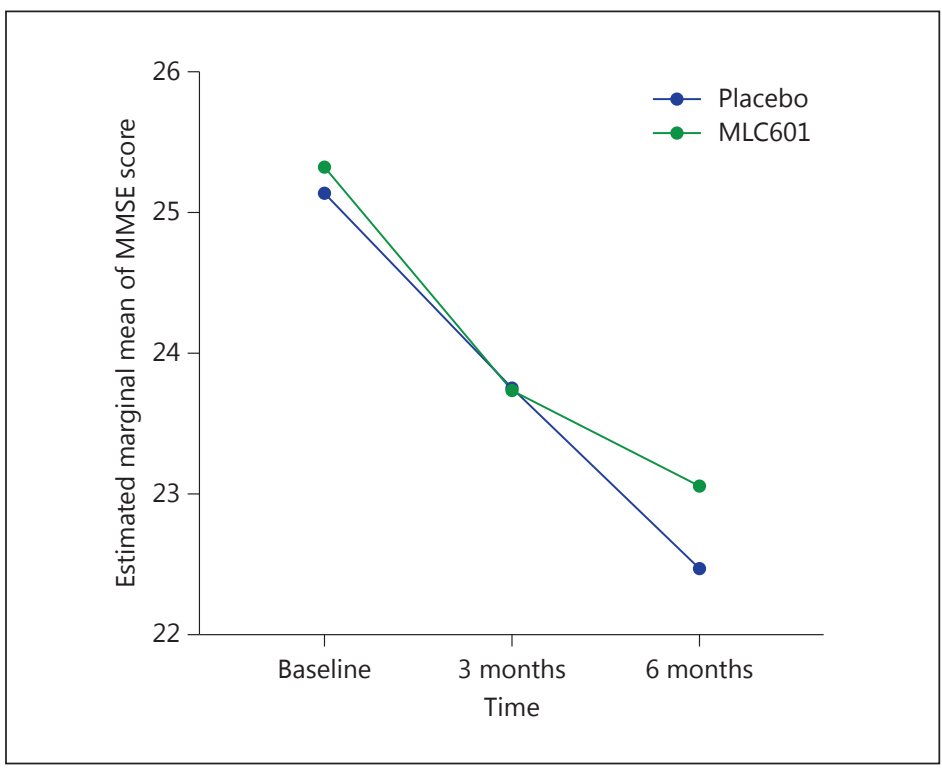

\begin{tabular}{llll}
\hline Group & Baseline & 3 months & 6 months \\
\hline MLC601 & $15.55 \pm 3.78$ & $17.20 \pm 5.80$ & $19.38 \pm 7.86$ \\
Placebo & $15.97 \pm 2.41$ & $17.80 \pm 3.44$ & $20.38 \pm 6.68$ \\
\hline
\end{tabular}

MLC601 group was $3.82( \pm 6.16)$, and for the placebo group it was $4.41( \pm 6.66)$. By the general linear model, repeated-measures analysis was carried out based on the ADAS-cog score, and it showed a statistically significant difference between the placebo and the MLC601 group during the 6 months of follow-up ( $p<0.001$ ) (Fig. 3).

\section{Safety Outcome}

Only 5 patients $(14.7 \%)$ reported AEs in the MLC601 group, including nausea (8.8\%), diarrhea (8.8\%), constipation (5.8\%), abdominal discomfort (2.9\%), and anorexia. Also, we did not find any abnormalities in blood count and in liver and renal function.

\section{Discussion}

MCI is a condition that may progress to dementia; therefore, it needs close observation, and in the long term it might necessitate medical therapy. To the best of our knowledge there still is a serious lack of drugs that could be used to slow its progression to more severe cognitive decline or dementia. MLC601 is a natural neuroprotective medication that could be used in the treatment of MCI considering its potential efficacy and few AEs. Our primary finding in this study is that there was a statistically significantly slower decline in cognitive function in patients of the MLC601 group when compared to those treated with placebo after 6 months. Also, the most predominant minor AEs of MLC601 treatment in our study were gastrointestinal symptoms, most commonly nausea and diarrhea, and no patient left our study due to AEs. 
Fig. 3. Mean changes in ADAS-cog score from baseline in the placebo and the MLC601 group.

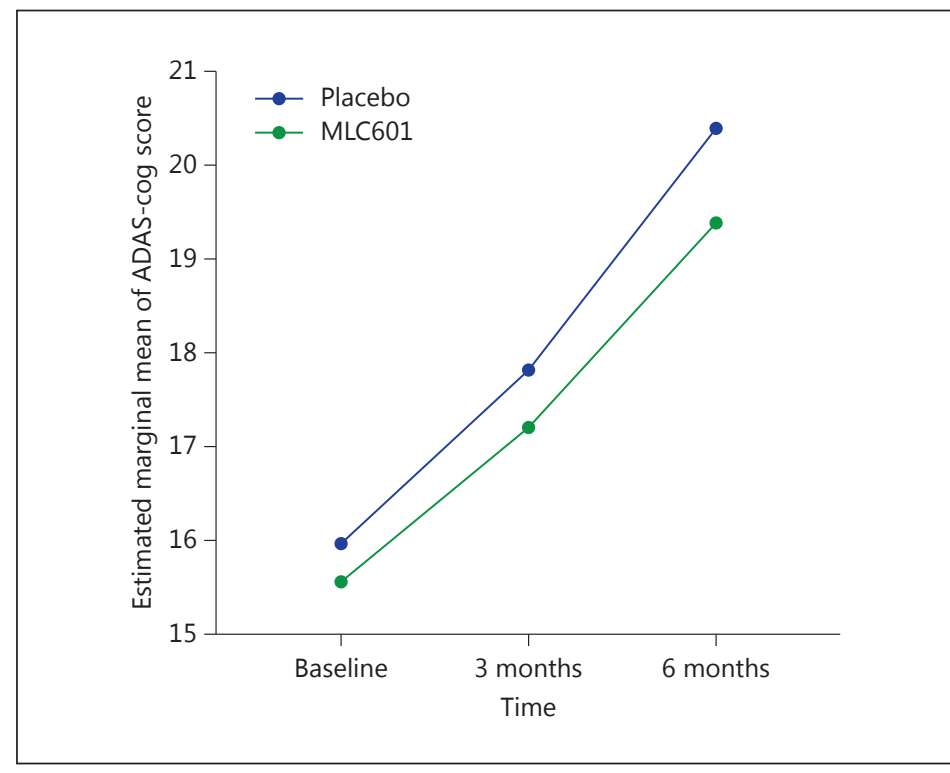

At the date of its publication, this has been the first clinical trial that evaluated early treatment of MCI patients with MLC601, and it showed a possible efficacy with acceptable tolerability. MLC601 has shown both neuroprotective and neuroregenerative properties in several controlled studies of ischemic injury to the brain [15-17]. It has been shown that MLC601 is a possible modulator of amyloid precursor protein processing and has implications as a putative therapeutic strategy for the treatment of post-stroke dementia and Alzheimer disease [18]. In our recent study [7], we compared the efficacy and safety of MLC601 with cholinesterase inhibitors. In that clinical trial study, MLC601 showed favorable tolerability and encouraging effects on cognitive function in Alzheimer disease patients during 18 months of treatment when compared to rivastigmine. In another study, MLC601 has shown a promising safety profile and also efficacy compared to the 3 FDA-approved cholinesterase inhibitors donepezil, rivastigmine, and galantamine. The study concluded that the trend for cognitive changes based on modifications over time in MMSE and ADAS-cog scores did not differ significantly between the groups ( $p=0.92$ for MMSE; $p=0.87$ for ADAS$\operatorname{cog}$ ) [8].

The safety of treatment with MLC601 was also noted in other studies; as in our study, the most commonly reported AEs were gastrointestinal, and they were transient and tolerable $[7,8,17,19]$. A difficulty with treating $\mathrm{MCI}$ is that some patients proceed to developing Alzheimer disease, while others do not and may even get better. Therefore, even if it were known that treatment at a particular time would help (e.g., prescribing a cholinesterase inhibitor), it would be unclear which patients with MCI should be treated and which should not [20]. In this situation, prescribing a drug with an acceptable efficacy and the least adverse effect seems reasonable.

We realize that there are limitations to our study. We did not use PET scanning or biomarker testing to pick up preclinical cases of Alzheimer disease. In addition, other aspects of cognition were not evaluated, and we followed our patients only for a short period of time. It is worth mentioning that our findings show a noticeable decline in cognition which may not be in line with the definition of MCI. This trend for change in cognition might be due to annual conversion rates of $10-15 \%$ to Alzheimer disease, the small sample size, the potential risk of anxiety and depression among patients, the limitation of our cognition batteries identifying 
patients with mild and early-onset dementia, and the intention-to-treat analysis in our study. However, this report can be considered a pilot study for performing complementary studies in this setting. We suggest that studies should focus on the effect of MLC601 on different subtypes of MCI, and we also recommend that trials should be conducted on larger study populations and with longer durations of follow-up.

\section{Disclosure Statement}

All authors declare that they have no conflict of interest.

\section{References}

1 Gauthier S, Reisberg B, Zaudig M, Petersen RC, Ritchie K, Broich K, Belleville S, Brodaty H, Bennett D, Chertkow H, et al: Mild cognitive impairment. Lancet 2006;367:1262-1270.

-2 Mitchell AJ, Shiri-Feshki M: Rate of progression of mild cognitive impairment to dementia - meta-analysis of 41 robust inception cohort studies. Acta Psychiatr Scand 2009;119:252-265.

-3 Tondelli M, Bedin R, Chiari A, Molinari MA, Bonifacio G, Lelli N, Trenti T, Nichelli P: Role of cerebrospinal fluid biomarkers to predict conversion to dementia in patients with mild cognitive impairment: a clinical cohort study. Clin Chem Lab Med 2015;53:453-460.

4 American Psychiatric Association: Diagnostic and Statistical Manual of Mental Disorders (DSM- $5^{\circledR}$ ). Arlington, American Psychiatric Association, 2013.

5 Chong MS, Sahadevan S: Preclinical Alzheimer's disease: diagnosis and prediction of progression. Lancet Neurol 2005;4:576-579.

6 Geda YE, Nedelska Z: Mild cognitive impairment: a subset of minor neurocognitive disorder? Am J Geriatr Psychiatry 2012;20:821-826.

-7 Harandi AA, Ashrafi F, Tabatabaei M, Ghorbani A, Hatamian H, Pakdaman H, Ilkhani M, Gharagozli K, Shojae M, Asadollahi M, et al: Efficacy and tolerability of MLC601 in patients with mild to moderate Alzheimer disease who were unable to tolerate or failed to benefit from treatment with rivastigmine. Br J Med Med Res 2013;3: 341-350.

-8 Pakdaman H, Harandi AA, Hatamian H, Tabatabae M, Delavar Kasmaei H, Ghassemi A, Gharagozli K, Ashrafi F, Emami Naeini P, Tavakolian M, Shahin D: Effectiveness and safety of MLC601 in the treatment of mild to moderate Alzheimer's disease: a multicenter, randomized controlled trial. Dement Geriatr Cogn Disord Extra 2015;5:96-106.

-9 Folstein MF, Folstein SE, McHugh PR: "Mini-mental state": a practical method for grading the cognitive state of patients for the clinician. J Psychiatr Res 1975;12:189-198.

10 Weyer G, Erzigkeit H, Kanowski S, Ihl R, Hadler D: Alzheimer's Disease Assessment Scale: reliability and validity in a multicenter clinical trial. Int Psychogeriatr 1997;9:123-138.

11 Petersen RC: Mild cognitive impairment as a diagnostic entity. J Intern Med 2004;256:183-194.

12 Petersen RC, Smith GE, Waring SC, Ivnik RJ, Tangalos EG, Kokmen E: Mild cognitive impairment: clinical characterization and outcome. Arch Neurol 1999;56:303-308.

-13 Rosen WG, Terry RD, Fuld PA, Katzman R, Peck A: Pathological verification of ischemic score in differentiation of dementias. Ann Neurol 1980;7:486-488.

14 Hamilton M: A rating scale for depression. J Neurol Neurosurg Psychiatry 1960;23:56-62.

15 Quintard H, Lorivel T, Gandin C, Lazdunski M, Heurteaux C: MLC901, a Traditional Chinese Medicine induces neuroprotective and neuroregenerative benefits after traumatic brain injury in rats. Neuroscience 2014;277: 72-86.

-16 Chen CL, Young SH, Gan HH, Singh R, Lao AY, Baroque AC 2nd, Chang HM, Hiyadan JHB, Chua CL, Advincula JM, et al: Chinese medicine NeuroAiD efficacy on stroke recovery: a double-blind, placebo-controlled, randomized study. Stroke 2013;44:2093-2100.

17 Siddiqui FJ, Venketasubramanian N, Chan ES-Y, Chen C: Efficacy and safety of MLC601 (NeuroAiD ${ }^{\circledR}$ ), a traditional Chinese medicine, in poststroke recovery: a systematic review. Cerebrovasc Dis 2013;35(suppl 1):8-17.

18 Lim YA, Murray LA, Lai MK, Chen C: NeuroAiD ${ }^{\circledR}$ (MLC601) and amyloid precursor protein processing. Cerebrovasc Dis 2013;35(suppl 1):30-37.

19 Harandi AA, Abolfazli R, Hatemian A, Ghragozlee K, Ghaffar-Pour M, Karimi M, Shahbegi S, Pakdaman H, Tabasi M, Tabatabae AL, Nourian A: Safety and efficacy of MLC601 in Iranian patients after stroke: a double-blind, placebo-controlled clinical trial. Stroke Res Treat 2011;2011:721613.

20 Howe E: Initial screening of patients for Alzheimer's disease and minimal cognitive impairment. Psychiatry (Edgmont) 2007;4:24-27. 